\title{
Is There Still a Place for Post-Resuscitation Electrocardiogram for Therapeutic Management and Coronary Angiogram Indication after Out-of-Hospital Cardiac Arrest?
}

\author{
Clément Lonjon ${ }^{*}$, Benoit Lattuca ${ }^{1 *}$, François Roubille1, Guillaume Cayla², \\ Florence Leclercq ${ }^{1 \#}$ \\ ${ }^{1}$ Cardiology Department, Arnaud de Villeneuve University Hospital, Montpellier, France \\ ${ }^{2}$ Cardiology Department, Caremeau University Hospital, Nimes, France \\ Email:
}

Received 4 July 2015; accepted 18 August 2015; published 21 August 2015

Copyright (C) 2015 by authors and Scientific Research Publishing Inc.

This work is licensed under the Creative Commons Attribution International License (CC BY).

http://creativecommons.org/licenses/by/4.0/

(c) (i) Open Access

\begin{abstract}
Out-of-hospital cardiac arrest is a common cause of death. Some therapeutic strategies performed daily are still debated, including particularly emergency coronary angiography independently of the clinical pattern. Primary percutaneous coronary intervention seems the strategy of choice in ST-segment elevation myocardial infarction but in other clinical presentations, benefit of coronary angiogram remains controversial. To improve management of out-of-hospital cardiac arrest and define the best timing to perform coronary angiogram, we suggest a study design based on ECG evaluation to define predictors of coronary artery disease after resuscitated cardiac arrest by distinguishing 3 groups according to ECG after resuscitation: ST segment elevation and LBBB; repolarisation disorder or no repolarisation disorder. Evaluation of ECG changes may still be useful as a triage method for establishing the indication for emergency coronary angiogram due to easy, non invasive and quick method and thus for limiting complications associated with this exam in acute phase.
\end{abstract}

\section{Keywords}

Coronary Angiogram, Cardiac Arrest, Resuscitation, STEMI, ECG Changes, Predictors

\footnotetext{
*Authors have equally contributed to this paper.

${ }^{\sharp}$ Corresponding author.
} 


\section{Introduction}

Out-of-hospital cardiac arrest (OHCA) is a common cause of death. The very low successful rates of resuscitation make sudden cardiac death (SCD) a major unsolved problem in clinical cardiology, emergency medicine, and public health. Each year, in United States, about 225,000 SCD are reported with an 8.4\% estimated survival rate [1] [2]. Likewise, in Europe, number of sudden death is estimated at 275,000 people per year with a $10 \%$ survival rate [3]. While there is currently no large study available concerning incidence and etiology of OHCA, data obtained from different registries and small studies are heterogeneous related to variable inclusion criteria [4]-[7]. In France, data were mainly obtained from the PROCAT registry (Parisian Region out of Hospital Cardiac arrest) and the national registry REAC [8] and the incidence of sudden deaths reported was between 40,000 to 50,000 each year.

According to the guidelines [9] [10], the management of OHCA is currently invasive and interventional during both pre- and in-hospital care. However, some therapeutic strategies performed daily are still debated, including particularly the use of systematic hypothermia or emergency coronary angiography independently of the clinical pattern.

Hypothermia induced indications and methods, especially concerning the target temperature, are the subject of many current studies and controversies after OHCA [11]-[13].

While coronary angiogram is mandatory in patients with resuscitated cardiac arrest and ST-segment elevation myocardial infarction (STEMI), current guidelines considered indications (class IIa Level B) of immediate angiography and possible primary Percutaneous Coronary Intervention (PCI) in survivors of cardiac arrest without ST-segment elevation on ECG but with a high suspicion of ongoing infarction [10]. Indeed, many clinical and post-mortem studies showed that the majority of OHCA was related to coronary artery disease (CAD) [14]-[17] but benefit of coronary angiogram in non selected OHCA remains controversial. Therefore, guidelines are currently based on the results of one study included only 9 patients with coronary occlusion without STEMI [16].

Subsequently, many small studies have been performed with relatively disparate results. Anyfantakis et al. showed that ST-segment elevation on admission was an independent predictor of acute myocardial infarction with high positive (82.6\%) and negative (83.7\%) predictive values [4]. In the study published by Sideris et al., combination of ST-segment elevation and/or depression was an excellent predictor of acute myocardial infarction associated to OHCA with a high negative predictive value (96\%). A combined criterion comprising STelevation and/or depression and/or left bundle branch block (LBBB) and/or non-specific wide QRS even shows a negative predictive value of $100 \%$ [18]. However, the study of Dumas et al. made from the PROCAT registry showed a much smaller negative predictive values of around 42\% [5].

Consequently, considering both the lack of data and controversial studies, indication of coronary angiogram is not clearly defined in OHCA except in obvious STEMI context where efficacy and reduction in mortality have been demonstrated with primary PCI [9].

Coronary angiogram remains an invasive procedure with potential adverse effects such as bleeding, particularly at punction site, renal insufficiency, stroke, coronary dissection, rupture or occlusion. Furthermore, unnecessary coronary angiogram may be associated with a potential waste of time and delay of specialized and intensive management. To limit unnecessary and even harmful testing, it can be useful to evaluate to benefit/risk balance of coronary angiogram, in a population of patients with OHCA, with exclusion of evident non cardiac cause of cardiac arrest. Out of STEMI setting with typical ECG changes, detection of predictors of significant coronary lesions potentially implicated in initial cardiac arrest may be useful in order to guide indication and time (immediate vs delayed) of coronary angiogram.

We therefore suggested the CASCAD study (Is Coronary Angiogram Systematic after Cardiac Arrest resuscitateD?) with the main objective to evaluate indication and best delay to perform coronary angiogram after a resuscitated cardiac arrest.

\section{Study Hypothesis}

Although the diagnostic value of the ST-segment elevation is uncertain in the OHCA setting, we hypothesised that ECG changes may still be useful as a triage method for establishing the indication for emergency coronary angiogram. Thus, we defined 3 groups of patients: ST segment elevation, repolarisation disorders without ST segment elevation and normal ECG. 


\section{Methods}

This study will be performed in 2 interventional university hospitals of Montpellier and Nimes (France), with collaboration of emergency mobile units and regulation.

Each interventional center has a 24/24 hours and 7/7 days available catheterization lab and specialized intensive care units where patients could be treated after successful resuscitation.

\subsection{Study Population and Patient Selection}

All consecutive patients admitted in catheterisation lab after resuscitated cardiac arrest will be eligible to this prospective observational registry.

\subsubsection{Inclusion Criteria}

- Patients aged $\geq 18$ years.

- Resuscitated OHCA and admission in catheterization lab for coronary angiogram whatever initial ECG.

\subsubsection{Exclusion Criteria}

- Patients with an obvious non-cardiac cause of OHCA.

- OHCA complicating acute disease diagnosed before the cardiac arrest.

- Contra-indication to coronary angiogram for medical or ethical implications.

\subsubsection{Data Collection}

Clinical and laboratory data will be obtained from hospital reports, electronic medical records and laboratory reports. All available ECG will be collected and analyzed by two independent experts to define the 3 groups of patients. All angiographic data will be collected and all examinations will be reviewed by 2 experts in interventional cardiology in a double blinded manner. In case of disagreement, a third expert opinion will be requested.

All data collected are represented in Table 1.

\subsection{Study Design and Experimental Protocol}

For this study, 3 groups were defined in according to ECG after resuscitation:

\section{- Groupe 1: ST segment elevation and LBBB}

ST-segment elevation is considered significant if present in two contiguous leads with the cut-points: $\geq 0.1$ $\mathrm{mV}$ in all leads other than leads V2-V3 where the following cut points apply: $\geq 0.2 \mathrm{mV}$ in men $\geq 40$ years; $\geq 0.25$ $\mathrm{mV}$ in men $<40$ years, or $\geq 0.15 \mathrm{mV}$ in women [19]. LBBB is defined as QRS duration $>120 \mathrm{~ms}$ with QS or $\mathrm{rS}$ pattern in V1 and broad R waves in lead L, V5 and V6 [20].

\section{- Groupe 2: repolarisation disorder (ST segment depression, $\mathrm{T}$ waves changes)}

ST segment depression is considered significant if $\geq 0.05 \mathrm{mV}$ in two contiguous leads [19]. $\mathrm{T}$ waves changes are defined by $\mathrm{T}$ inversion $\geq 0.1 \mathrm{mV}$ in two contiguous leads [19].

- Groupe 3: no repolarisation disorder: (normal ECG, RBBB without other significant changes, isolated atrioventricular block, supraventricular arrhythmia without other significant changes)

RBBB is defined as QRS duration >120 ms with rSR' complex in V1 V2 and S wave in lead L and and V5 V6. Angiographic results will be analyzed in each group to evaluate the incidence of coronary stenosis and/or occlusion potentially involved in the cardiac arrest and to define predictive factors of acute CAD.

The study design is represented in Figure 1.

\subsection{Perspectives}

According to the results of this first study, we plan to perform a multicentric randomized trial testing immediate coronary angiogram versus delayed coronary angiogram in patients selected according to ECG patterns. This study protocol could be represented as in Figure 2.

\section{Discussion}

Despite very low data, primary PCI seems the strategy of choice in STEMI complicated by OHCA with probable 


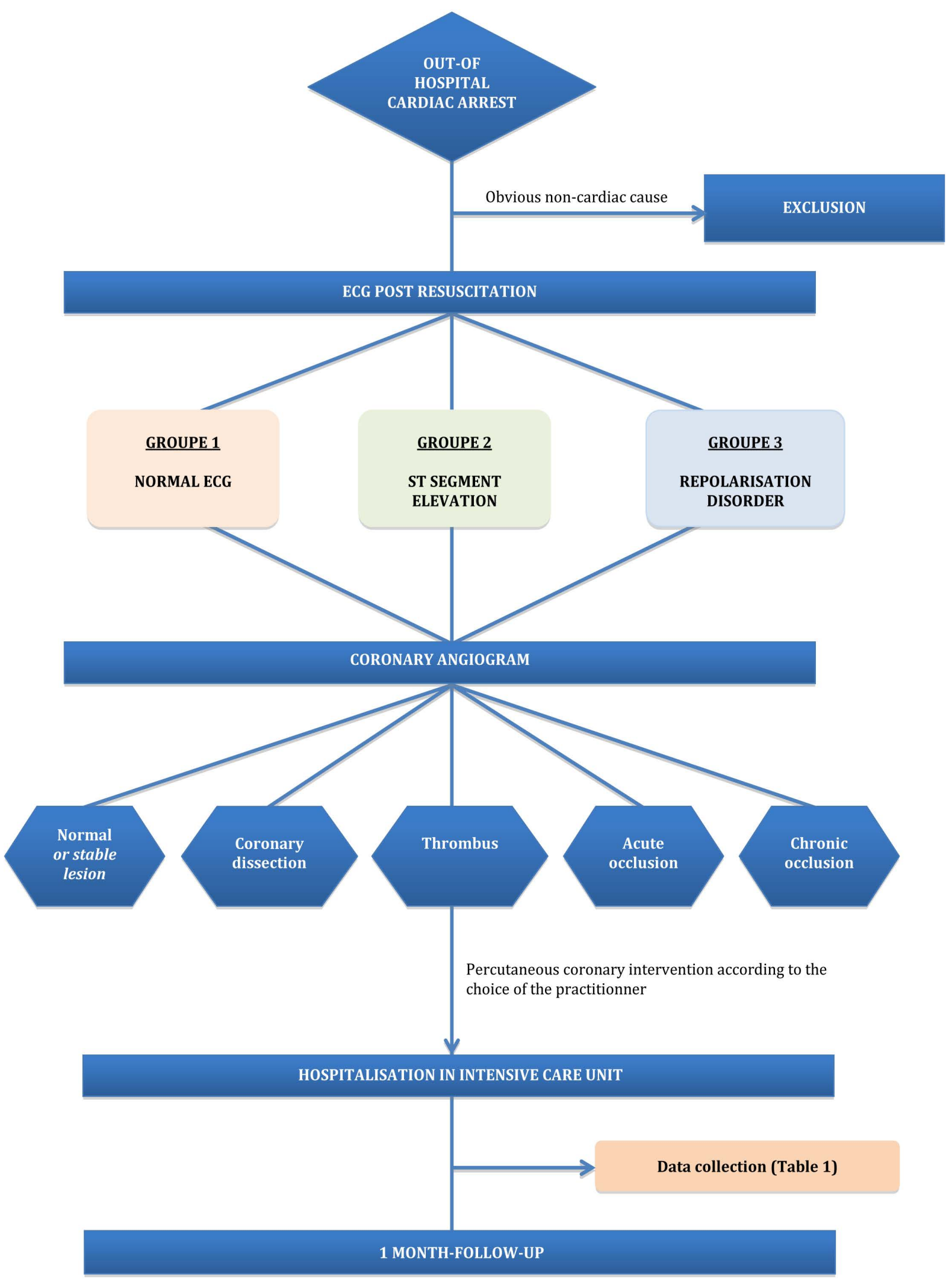

Figure 1. Study design and experimental protocol. 


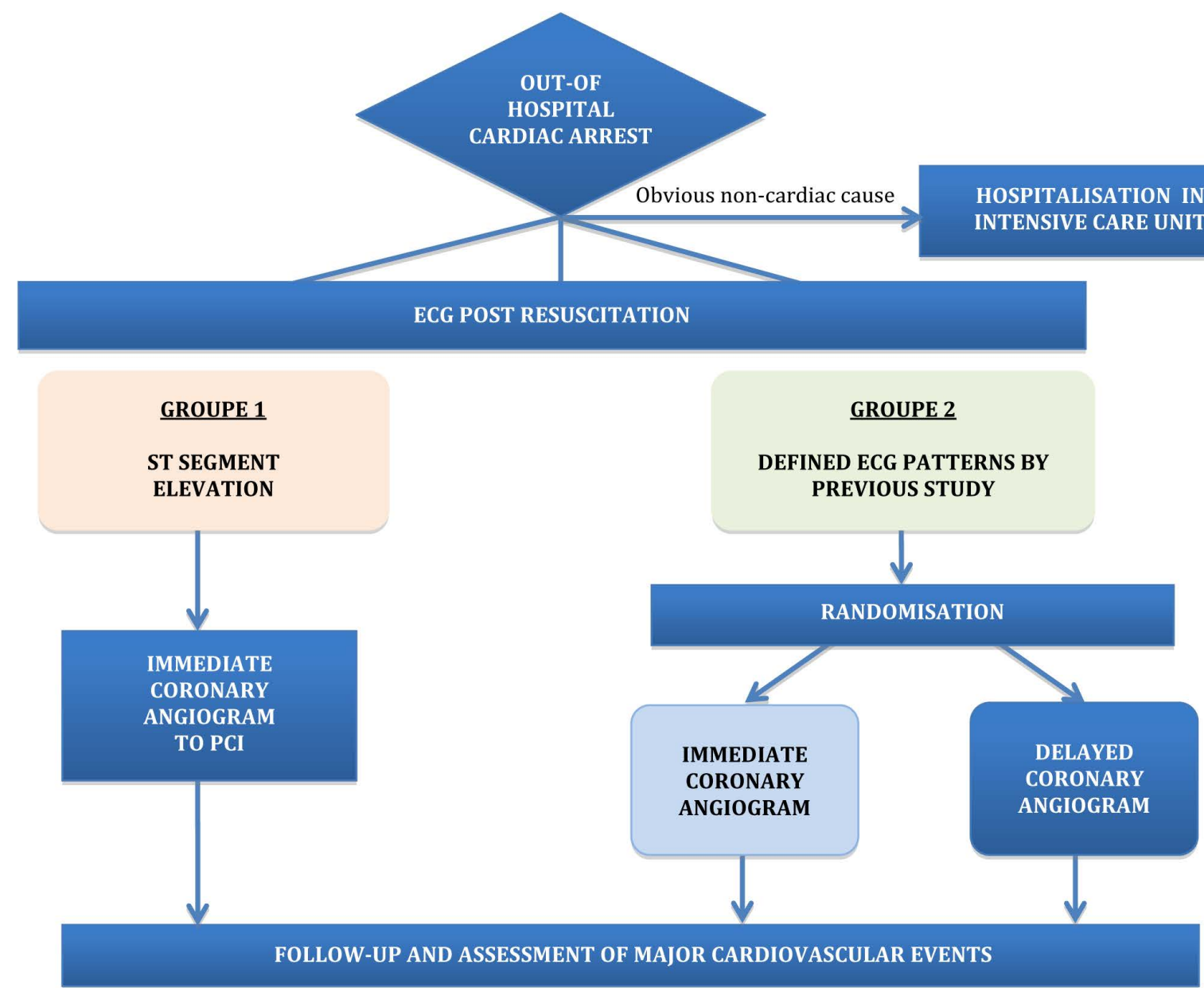

Figure 2. Study design of suggested randomised study.

Table 1. Study data collection.

\begin{tabular}{|c|c|}
\hline & Clinical Pattern \\
\hline Cardiovascular risk factors & $\begin{array}{ll}\text { - } & \text { Age } \\
\text { - } & \text { Sex } \\
\text { - } & \text { BMI } \\
\text { - } & \text { Smoking } \\
\text { - High blood pressure } \\
\text { - } \\
\text { - } & \text { Diablipidemia } \\
\end{array}$ \\
\hline Medical history & $\begin{array}{l}\text { - Coronary artery disease (medical, percutaneous Coronary } \\
\text { intervention or coronary bypass) } \\
\text { - Dilated cardiomyopathy } \\
\text { - Chronic obstructive pulmonary disease }\end{array}$ \\
\hline Out of hospital data & $\begin{array}{l}\text { - Time of no flow } \\
\text { - Time of low flow } \\
\text { - } \text { Duration of cardiac arrest } \\
\text { - Use of external cardiac shock } \\
\text { - Use and dose of adrenaline }\end{array}$ \\
\hline Biological data & $\begin{array}{l}\text { - Peak of ultra-sensitive Troponin } \\
\text { - } \quad \text { Lactic acid } \\
\text { - } \text { Creatinine } \\
\text { Hepatic markers and total bilirubin }\end{array}$ \\
\hline
\end{tabular}




\section{Continued}

\begin{tabular}{|c|c|}
\hline Follow-up data & $\begin{array}{l}\text { - Death and cause of death } \\
\text { - Cause of cardiac arrest } \\
\text { - Complications of resuscitation and hospitalisation in } \\
\text { - intensive care unit } \\
\text { - Automatic defibrillator implantation }\end{array}$ \\
\hline \multicolumn{2}{|r|}{ Electrocardiogram Data } \\
\hline Initial ECG rhythm & $\begin{array}{l}\text { - } \text { - } \text { Asystricular tachycardia or fibrillation } \\
\text { - } \text { Pulseless electrical activity } \\
\text { - } \text { Torsades de pointe } \\
\text { - Atrioventricular block } \\
\text { - Not determined shockable rhythm }\end{array}$ \\
\hline ECG after resuscitation & $\begin{array}{ll}\text { - } & \text { ST-segment elevation } \\
\text { - } & \text { ST-segment depression } \\
\text { - } & \text { Left Branch Bundle Block } \\
\text { - } & \text { Right Branch Bundle Block } \\
\text { - } & \text { Atypical segment ST modification } \\
\text { - } & \text { Normal ECG }\end{array}$ \\
\hline
\end{tabular}

\section{Angiographic Data}

- Normal

- Not significant atheroma

Coronary angiogram evaluation

- Single-vessel disease

- Double-vessel disease

- Triple-vessel disease

- Acute coronary occlusion

- Chronic coronary occlusion

Type of lesion

- Thombus

- Coronary dissection

- Stable lesion

- Left main

Culprit coronary lesion

- Left anterior descending ( LAD )

- Circumflex ( CX )

- Right coronary artery ( RCA )

TIMI classification

- Before and after procedure

Coronary stents

Ventriculography evaluation

- $\quad$ Type and number

- Normal Left Ventricular Ejection Fraction (LVEF)

- Global hypokinesia

- Segmental disorders

- Use of transient electrosystolic pacing

- $\mathrm{CO}_{2}$ expired

Others procedures

- Use of Intra-Aortic counterpulsation

- Use of extracorporeal life support system

- Use of Impella system

Echocardiographic Assessment

- Normal LVEF

- Global hypokinesia

- Segmental disorders

- Left ventricle hypertrophy

Right ventricule

- Right ventricle dilatation

- Right ventricle dysfunction

Pericardium

- Effusion 
positive risk/benefit balance in small studies [21] [22]. In the absence of ST segment elevation, coronary angiogram is widely recommended and guidelines are only based on an initial study published in 1997 by Spaulding et al. showing that absence of ST segment elevation was not predictive of absence of culprit coronary lesions. In this study, only 9 patients had coronary occlusions without STEMI. However, the study design and low population did not suggest a relationship between coronary damage and cardiac arrest in patients with multiple cardiovascular risk factors and a high prevalence of CAD.

Indeed, ECG changes may still be useful as a triage method for establishing the indication for emergency coronary angiogram due to easy, non invasive and quick method. It seems necessary to evaluate predictors of CAD after resuscitated cardiac arrest to improve management of patients and diagnosis procedures. Group of patients who really benefit from emergency coronary angiogram must be defined and evaluation of ECG after resuscitation could be an appropriate method although further investigations are required.

\section{References}

[1] Eisenberg, M.S. and Mengert, T.J. (2001) Cardiac Resuscitation. New England Journal of Medicine, 344, $1304-1313$. http://dx.doi.org/10.1056/NEJM200104263441707

[2] Rea, T.D., Eisenberg, M.S., Sinibaldi, G. and White, R.D. (2004) Incidence of EMS-Treated Out-of-Hospital Cardiac Arrest in the United States. Resuscitation, 63, 17-24. http://dx.doi.org/10.1016/j.resuscitation.2004.03.025

[3] Atwood, C., Eisenberg, M.S., Herlitz, J. and Rea, T.D. (2005) Incidence of EMS-Treated Out-of-Hospital Cardiac Arrest in Europe. Resuscitation, 67, 75-80. http://dx.doi.org/10.1016/j.resuscitation.2005.03.021

[4] Anyfantakis, Z.A., Baron, G., Aubry, P., Himbert, D., Feldman, L.J., Juliard, J.-M., et al. (2009) Acute Coronary Angiographic Findings in Survivors of Out-of-Hospital Cardiac Arrest. American Heart Journal, 157, 312-318. http://dx.doi.org/10.1016/j.ahj.2008.09.016

[5] Dumas, F., White, L., Stubbs, B.A., Cariou, A. and Rea, T.D. (2012) Long-Term Prognosis Following Resuscitation from Out of Hospital Cardiac Arrest: Role of Percutaneous Coronary Intervention and Therapeutic Hypothermia. Journal of the American College of Cardiology, 60, 21-27. http://dx.doi.org/10.1016/j.jacc.2012.03.036

[6] Dumas, F., Cariou, A., Manzo-Silberman, S., Grimaldi, D., Vivien, B., Rosencher, J., et al. (2010) Immediate Percutaneous Coronary Intervention Is Associated with Better Survival after Out-of-Hospital Cardiac Arrest: Insights from the PROCAT (Parisian Region Out of hospital Cardiac ArresT) Registry. Circulation: Cardiovascular Interventions, 3, 200-207. http://dx.doi.org/10.1161/CIRCINTERVENTIONS.109.913665

[7] Spaulding, C.M., Joly, L.M., Rosenberg, A., Monchi, M., Weber, S.N., Dhainaut, J.F., et al. (1997) Immediate Coronary Angiography in Survivors of Out-of-Hospital Cardiac Arrest. New England Journal of Medicine, 336, 1629-1633. http://dx.doi.org/10.1056/NEJM199706053362302

[8] RéAC, Registre électronique des Arrêts Cardiaques. http://registreac.org/

[9] 2014 ESC/EACTS. Guidelines in Myocardial Revascularisation (Guidelines for). http://www.escardio.org/Guidelines-\&-Education/Clinical-Practice-Guidelines/ESC-EACTS-Guidelines-in-Myocardial -Revascularisation-Guidelines-for

[10] (2012) ESC Guidelines for the Management of Acute Myocardial Infarction in Patients Presenting with ST-Segment Elevation. European Heart Journal, 33, 2569-2619. http://dx.doi.org/10.1093/eurheartj/ehs215

[11] Bernard, S.A., Gray, T.W., Buist, M.D., Jones, B.M., Silvester, W., Gutteridge, G., et al. (2002) Treatment of Comatose Survivors of Out-of-Hospital Cardiac Arrest with Induced Hypothermia. New England Journal of Medicine, 346, 557-563. http://dx.doi.org/10.1056/NEJMoa003289

[12] Clinkard, D., Cameron, A., Howes, D. and Ball, I. (2015) Targeted Temperature Management: It Is Not Yet Time to Change Your Target Temperature. CJEM, 1-3. http://dx.doi.org/10.1017/cem.2014.35

[13] Belliard, G., Catez, E., Charron, C., Caille, V., Aegerter, P., Dubourg, O., et al. (2007) Efficacy of Therapeutic Hypothermia after Out-of-Hospital Cardiac Arrest Due to Ventricular Fibrillation. Resuscitation, 75, 252-259. http://dx.doi.org/10.1016/j.resuscitation.2007.04.014

[14] Zheng, Z.J., Croft, J.B., Giles, W.H. and Mensah, G.A. (2001) Sudden Cardiac Death in the United States, 1989 to 1998. Circulation, 104, 2158-2163. http://dx.doi.org/10.1161/hc4301.098254

[15] Zipes, D.P. and Wellens, H.J.J. (1998) Sudden Cardiac Death. Circulation, 98, 2334-2351. http://dx.doi.org/10.1161/01.CIR.98.21.2334

[16] Pell, J.P., Sirel, J.M., Marsden, A.K., Ford, I., Walker, N.L. and Cobbe, S.M. (2003) Presentation, Management, and Outcome of Out of Hospital Cardiopulmonary Arrest: Comparison by Underlying Aetiology. Heart, 89, 839-842. http://dx.doi.org/10.1136/heart.89.8.839 
[17] Davies, M.J. (1992) Anatomic Features in Victims of Sudden Coronary Death. Coronary Artery Pathology. Circulation, 85, I19-I24.

[18] Sideris, G., Voicu, S., Dillinger, J.G., Stratiev, V., Logeart, D., Broche, C., et al. (2011) Value of Post-Resuscitation Electrocardiogram in the Diagnosis of Acute Myocardial Infarction in Out-of-Hospital Cardiac Arrest Patients. Resuscitation, 82, 1148-1153. http://dx.doi.org/10.1016/j.resuscitation.2011.04.023

[19] Thygesen, K., Alpert, J.S., Jaffe, A.S., Simoons, M.L., Chaitman, B.R., White, H.D., et al. (2012) Third Universal Definition of Myocardial Infarction. Global Heart, 7, 275-295. http://dx.doi.org/10.1016/j.gheart.2012.08.001

[20] Menown, I.B., Mackenzie, G. and Adgey, A.A. (2000) Optimizing the Initial 12-Lead Electrocardiographic Diagnosis of Acute Myocardial Infarction. European Heart Journal, 21, 275-283. http://dx.doi.org/10.1053/euhj.1999.1748

[21] Kahn, J.K., Glazier, S., Swor, R., Savas, V. and O’Neill, W.W. (1995) Primary Coronary Angioplasty for Acute Myocardial Infarction Complicated by Out-of-Hospital Cardiac Arrest. The American Journal of Cardiology, 75, 10691070. http://dx.doi.org/10.1016/S0002-9149(99)80727-9

[22] Bendz, B., Eritsland, J., Nakstad, A.R., Brekke, M., Kløw, N.E., Steen, P.A., et al. (2004) Long-Term Prognosis after Out-of-Hospital Cardiac Arrest and Primary Percutaneous Coronary Intervention. Resuscitation, 63, 49-53. http://dx.doi.org/10.1016/j.resuscitation.2004.04.006 